\title{
Accelerated genetic algorithms with Markov Chains
}

\author{
Guan Wang*, Chen Chen**, and K. Y. Szeto*** \\ Department of Physics, the Hong Kong University of Science and Technology \\ Hong Kong, China
}

\begin{abstract}
[Abstract] Based on the mutation matrix formalism and past statistics of genetic algorithm, a Markov Chain transition probability matrix is introduced to provide a guided search for complex problem optimization. The important input for this guided search is the ranking scheme of the chromosomes. It is found that the effect of mutation using the transition matrix yields faster convergence as well as overall higher fitness in the search for optimal solutions for the $0-1$ Knapsack problem, when compared with the mutation-only-genetic-algorithm, which include the traditional genetic algorithm as a special case. The accelerated genetic algorithm with Markov Chain provides a theoretical basis for further mathematical analysis of evolutionary computation, specifically in the context of adaptive parameter control.
\end{abstract}

Key words: genetic algorithm, Markov Chains, Optimization, Knapsack

\section{Introduction}

Successful applications of genetic algorithm using the Darwinian principle of survival of the fittest have been implemented in many areas [1,2], such as in solving the crypto-arithmetic problem [3], time series forecasting [4], traveling salesman problem [5], function optimization [6], adaptive agents in stock markets [7,8], and airport scheduling [9]. A drawback for the practitioners of genetic algorithm is the need for expertise in the specific application, as its efficiency depends very much on the parameters chosen in the evolutionary process. An example of this drawback can be found in the ad-hoc manner in choosing the selection mechanism, where different percentages of the population for survival for different problems are used. Since the parameters used in a specific application will generally be suboptimal in a different application, the traditional usage of genetic algorithm is more like an artisan approach. To tackle this shortcoming, we attempt an

*Present address of Guan Wang is the Department of Physics, Peking University, Beijing, China

** Present address of Chen Chen, Columbia University, New York, USA

***Corresponding author’s email: phszeto@ust.hk 
adaptive approach where the number of parameters requiring input from experts is reduced to a minimum. The basic aim is to make use of past data collected in the evolutionary process of genetic algorithm to tune the parameters such as the survival probability of a chromosome. We can collect the statistics of the chromosomes in past generations to decide the probability of mutation of each locus $[10,11]$. A general formalism using this idea has been constructed through the time dependent mutation matrix, and the algorithm called MOGA (mutation only genetic algorithm) has been shown to be very successful in solving various practical problems[3-9]. Generalization of this formalism to include crossover has also been developed in a recent publication [12]. This approach is reviewed briefly in Section 2.

The objective of this paper is to present a more systematic way of making use of the statistic of the locus during evolution to provide a guided search in optimization. Similar to MOGA discussed in Section 2, the mutation probability is time dependent. However, the dependence is not based on the tensor product of the chromosome mutation probability and locus mutation probability. We make use a Markov chain to describe the evolution of the probability of mutation, or more generally, the transition probability between 0 and 1 state in a binary encoding of the chromosomes in the genetic algorithm. The entire approach is based on the existence of a target state provided by the fit chromosomes, with proper weighting. In this approach, which we called MCGA (Markov Chain Genetic Algorithm), the really important input of this algorithm is the weighting function applied for the chromosomes, which is defined by the fitness ranking scheme. We will develop this algorithm in section 3. We compare this MCGA with our previous MOGA algorithm using the standard 0-1 knapsack problem in section 4. Even though this comparison is limited to the knapsack problem, we expect this approach of MCGA is more flexible for further development. We will discuss these issues at the end of the paper.

\section{Mutation Matrix and MOGA}

In traditional simple genetic algorithm, the mutation/crossover operators are processed on the chromosome indiscriminately over the loci without making use of the loci statistics, which has been demonstrated to provide useful information on mutation operator [12]. In our mutation matrix formalism, the traditional genetic algorithm can be treated as a special case. Let's consider a population of $\mathrm{N}$ chromosomes, each of length $\mathrm{L}$ and binary encoded. We describe the population by a $N \times L$ matrix, with entry $A_{i j}(t), i=1, \ldots, N ; j=1, \ldots, L$ denoting the value of the $j$ th locus of the $i$ th chromosome. The convention is to order the rows of $\mathrm{A}$ by the fitness of the chromosomes, $f_{i}(t) \leq f_{k}(t)$ for $i \geq k$. Traditionally we divide the population of $N$ chromosomes into three groups: (1) Survivors who are the fit ones. They form the first $N_{1}$ rows of the population matrix $A(t+1)$. Here $N_{1}=c_{1} N$ 
with the survival selection ratio $0<c_{1}<1$. (2) The number of children is $N_{2}=C_{2} N$ and is generated from the fit chromosomes by genetic operators such as mutation. Here $0<c_{2}<1-c_{1}$ is the second parameter of the model. We replace the next $N_{2}$ rows in the population matrix $A(t+1)$. $\quad$ (3) the remaining $N_{3}=N-N_{1}-N_{2}$ rows are the randomly generated chromosomes to ensure the diversity of the population so that the genetic algorithm continuously explores the solution space.

In our formalism, we introduce a mutation matrix with elements $M_{i j}(t) \equiv a_{i}(t) b_{j}(t), i=1, \ldots, N ; j=1, \ldots, L ; \quad 0 \leq a_{i}(t), b_{j}(t) \leq 1$ where $a_{i}(t) \quad$ and $\quad b_{j}(t)$ are called the row mutation probability and column mutation probability respectively. Traditional genetic algorithm with mutation as the only genetic operator corresponds to a time independent mutation matrix with elements $M_{i j}(t) \equiv 0$ for $i=1, \ldots, N_{1}, M_{i j}(t) \equiv m \in(0,1)$ for $i=N_{1}+1, \ldots, N_{2}$, and finally we have $M_{i j}(t) \equiv 1$ for $i=N_{2}+1, \ldots, N$. Here $m$ is the time independent mutation rate. We see that traditional genetic algorithm with mutation as the only genetic operator requires at least three parameters: $N_{1}, N_{2}$, and $m$.

We first consider the case of mutation on a fit chromosome. We expect to mutate only a few loci so that it keeps most of the information unchanged. This corresponds to "exploitation" of the features of fit chromosomes. On the other hand, when an unfit chromosome undergoes mutation, it should change many of its loci so that it can explore more regions of the solution space. This corresponds "exploration". Therefore, we require that $M_{i j}(t)$ should be a monotonic increasing function of the row index $i$ since we order the population in descending order of fitness. There are many ways to introduce the row mutation probability. One simple solution is to use $a_{i}(t)=(i-1) /(N-1)$. Next, we must decide on the choice of loci for mutation once we have selected a chromosome to undergo mutation. This is accomplished by computing the locus mutation probability of changing to $\mathrm{X}(\mathrm{X}=0$ or 1$)$ at locus $j$ as $p_{j X}$ by

$$
p_{j X}=\sum_{k=1}^{N}(N+1-k) \delta_{k j}(X) / \sum_{m=1}^{N} m
$$

Here $k$ is the rank of the chromosome in the population. $\delta_{k j}(X)=1$ if the $j$-th locus of the $k$-th chromosome assume the value $\mathrm{X}$, and zero otherwise. The factor in the denominator is for normalization. Note that $p_{j X}$ contains information of both locus and row and the locus statistics is biased so that heavier weight for chromosomes with high fitness is assumed. This is in general better than the original me- 
thod of Ma and Szeto[10] where there is no bias on the row. After defining $p_{j X}$, we define the column mutation rate as

$$
b_{j}=\left(1-\left|p_{j 0}-0.5\right|-\left|p_{j 1}-0.5\right|\right) / \sum_{j^{\prime}=1}^{L} b_{j^{\prime}}
$$

For example, if 0 and 1 are randomly distributed, we have $p_{j 0}=p_{j 1}=0.5$. There will be no useful information about the locus, so we should mutate this locus, and $b_{j}=1$. When there is definitive information, such as when $p_{j 0}=1-p_{j 1}=0$ or 1 , we should not mutate this column and we have $b_{j}=0$.

Once the mutation matrix $\mathbf{M}$ is obtained, we are ready to discuss the strategy of using $\mathbf{M}$ to evolve A. There are two ways to do Mutation Only Genetic Algorithm (MOGA). We can first decide which row (chromosome) to mutate, then which column (locus) to mutate, we call this particular method the Mutation Only Genetic Algorithm by Row or abbreviated as MOGAR. Alternatively, we can first select the column and then the row to perform mutation. We call this the Mutation Only Genetic Algorithm by Column or abbreviated as MOGAC. For MOGAR, we go through the population matrix A(t) by row first. The first step is to order the set of locus mutation probability $b_{j}(t)$ in descending order. This ordered set will be used for the determining of the set of column position (locus) in the mutation process. Now, for a given row $i$, we generate a random number $\mathrm{x}$. If $x<a_{i}(t)$, then we perform mutation on this row, otherwise we proceed to the next row and $A_{i j}(t+1)=A_{i j}(t), j=1, \ldots, L$. If row $i$ is to be mutated, we determine the set $R_{i}(t)$ of loci in row $i$ to be changed by choosing the loci with $b_{j}(t)$ in descending order, till we obtain $K_{i}(t)=a_{i}(t) * L$ members. Once the set $R_{i}(t)$ has been constructed, mutation will be performed on these columns of the $i$-th row of the A(t) matrix to obtain the matrix elements $A_{i j}(t+1), j=1, \ldots, L$. We then go through all $N$ rows, so that in one generation, we need to sort a list of $L$ probabilities $b_{j}(t)$ and generate $N$ random numbers for the rows. After we obtained $A(t+1)$, we need to compute the $M_{i j}(t+1)=a_{i} b_{j}(t+1)$ and proceed to the next generation.

For MOGAC, the operation is similar to MOGAR mathematically except now we rotate the matrix A by 90 degrees. Now, for a given column $j$ we generate a random number y. If $y<b_{j}(t)$, then we mutate this column, otherwise we proceed to the next column and $A_{i j}(t+1)=A_{i j}(t), i=1, \ldots, N$. If column $j$ is to be mutated, we determine the set $S_{j}(t)$ of chromosomes in column $j$ to be changed by choosing the rows with the $a_{i}(t)$ in descending order, till we obtain $W_{j}(t)=b_{j}(t) * N$ members. Since our matrix A is assumed to be row ordered by fitness, we simply need to choose the $N, N-1, \ldots, N-W_{j}+1$ rows to have the $j$-th 
column in these row mutated to obtain the matrix elements $A_{i j}(t+1), i=1, \ldots, N$ We then go through all $L$ columns, so that in one generation, we need to sort a list of $N$ fitness values and generate $L$ random numbers for the columns.

For a controlled comparison between MOGA and MCGA, we first choose which row (chromosome) to mutate, then which column (locus) to mutate. In this way, we can will compare MCGA with MOGAR on fair ground, since in our MCGA, we also decide which row to mutate before deciding which locus to change. The difference then is in the change of the locus for a given chromosome. In MOGAR, this change of the locus is governed by the probability $b(j)$, while in MCGA, this change is based on the transition matrix $\boldsymbol{P}$ for that particular locus $j$ at time $t$.

\section{Markov-Chain accelerated Genetic Algorithms}

In MOGA, the mutation matrix is obtained through the assignment of the row and column mutation probability, $M_{i j}(t) \equiv a_{i}(t) b_{j}(t), i=1, \ldots, N ; j=1, \ldots, L$. In the

implementation of the mutation, there are two different ways or ordering, MOGA by row or MOGA by column. However, both methods of implementation are rather ad-hoc. It will be desirable to implement the mutation on a more theoretical platform. Since we impose no memory effect, we should be able to describe the evolution process of the mutation probability through a Markov chain. Let us denote the population matrix by A. There are $N$ rows each representing a chromosome and $L$ columns each representing a locus. We assume that A is a binary matrix where every entry is either 0 or 1 . Let's assume that there is a transition probability between the population $\mathrm{A}(\mathrm{t})$ with $\mathrm{A}(\mathrm{t}+1)$ in the next generation. This transition should involve the basic features of genetic operators, which in the present paper concerns only the mutation process.

In the proposed genetic algorithm, we consider a mutation-only updating scheme between two generations. During each generation, chromosomes are sorted from the fittest one to the least fit one. The chromosome at the $i$-th fittest place is assigned a row mutation probability $a(i)$ according to some monotonic increasing function of its ranking: $r(i)=(i-1) / N^{\prime}$, if $i-1<N^{\prime}$ and $r(i)=1$, otherwise. Here we use $N^{\prime}=N / 2$. This choice of $r(i)$ defines the ranking scheme we used. We also define the survival probability as $s(i)=1-r(i)$. In this way, $a(i)$ and $s(i)$ together decide the probability to mutate to the other $0-1$ state or to remain in the current 0 - 1 state for the $i$-th fittest chromosome. In the spirit of "survival of the fittest", we can use $s(i)$ as the statistical weight of importance for the $i$-th chromosomes: $w(i)=s(i)$.

After discussing the row mutation, let's now turn to the column mutation, which addresses the relevance of the statistical importance of the locus. For each locus $j$, we define $C_{0}(j)$ and $C_{1}(j)$ which count in the current generation the numbers of chromosomes whose $j$-th entries are " 0 "s and " 1 "s. Next we normalize these counts to obtain 


$$
n_{o}(j, t)=\frac{C_{o}(j)}{C_{o}(j)+C_{1}(j)} ; \quad n_{1}(j, t)=\frac{C_{1}(j)}{C_{o}(j)+C_{1}(j)}
$$

The normalized vector $\vec{n}(j, t)=\left(n_{O}(j), n_{1}(j)\right)$ characterizes the state distribution of the $j$-th locus among all the chromosomes in the current generation at time t.

In order to direct the current population to a preferred state distribution for locus $j$, we first look at those rows of the population matrix A that has the $j$-th locus assuming the value $\boldsymbol{u}(=0$ or 1$)$. Among these rows with $j$-th locus equal to $\boldsymbol{u}$, let's assume that the row that has maximum weight is the $i^{*}$-th row, we should then follow the $i^{*}$ row as it is the fittest and the weight $\mathrm{w}\left(i^{*}\right)$ is largest among the $N$ rows. We can then rewrite

$$
\begin{aligned}
& C_{o}^{\prime}(j)=\max \left\{W(i) \mid A_{i j}=0 ; i=1, \ldots, N\right\} ; \\
& C_{1}^{\prime}(j)=\max \left\{W(i) \mid A_{i j}=1 ; i=1, \ldots, N\right\}
\end{aligned}
$$

with their normalized forms

$$
n_{o}(j, t+1)=\frac{C_{o}^{\prime}(j)}{C_{o}^{\prime}(j)+C_{1}^{\prime}(j)} ; \quad n_{1}(j, t+1)=\frac{C_{1}^{\prime}(j)}{C_{o}^{\prime}(j)+C_{1}^{\prime}(j)} .
$$

The vector $\vec{n}(j, t+1)=\left(n_{o}(j, t+1), n_{1}(j, t+1)\right)$ provides a direction that the population should evolve. This vector characterizes the target state distribution of the locus $j$ among all the chromosomes in the next generation. Note that in this definition of the target state, we make use of the weight $\{w(i)\}$ of the rows, which we intuitively set to be the survival probability of the chromosomes $\{s(i)\}$. In turn, the survival probability is $\{(1-r(i))\}$, which is the type of ranking $\{r(i)\}$ used in the formulation. A different scheme of fitness ranking of the chromosomes will produce a different set of $\{w(i)\}$, thereby a different direction vector $\{\vec{n}(j, t+1)\}$. Therefore, the ultimate tunable quantity in MCGA is our choice of ranking scheme for the chromosomes.

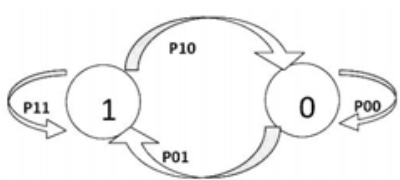

Fig.1. Markov-Chain with transition probability $P_{a b}$ from the current state $a$ to the state $b$. 
Assuming that we have chosen a monotonic ranking scheme for the fitness, and have worked out the direction vector for locus $j$, we can then apply Markov Chain theory to compute the transition probability of the various states as defined below

$$
\left(\begin{array}{l}
n_{o}(j, t+1) \\
n_{1}(j, t+1)
\end{array}\right)=\left(\begin{array}{ll}
P_{00}(j, t) & P_{10}(j, t) \\
P_{01}(j, t) & P_{11}(j, t)
\end{array}\right)\left(\begin{array}{l}
n_{o}(j, t) \\
n_{1}(j, t)
\end{array}\right)
$$

In the Markov Chain described in Fig.1, state 1 changes to state 0 with probability $P_{10}(j, t)$, and remains to be in state 1 with probability $P_{11}(j, t)=1-P_{10}(j, t)$. Similarly, state 0 changes to state 1 with probability $P_{01}(j, t)$, and remains to be state 0 with probability $P_{00}(j, t)=1-P_{01}(j, t)$. These conditions allow us to solve for the transition probability $P_{10}(j, t)$ explicitly in terms of the single variable $P_{00}(j, t)$ as

$$
\begin{aligned}
& P_{10}(j, t)=\frac{n_{o}(j, t+1)-P_{00}(j, t) n_{o}(j, t)}{n_{1}(j, t)} \\
& P_{11}(j, t)=1-P_{10}(j, t) \text { and } P_{01}(j, t)=1-P_{00}(j, t)
\end{aligned}
$$

Thus, we need only to know the probability $P_{00}(j, t)$ to compute all the remaining probabilities. At the beginning, we can set $P_{00}\left(j, t_{i n i}\right)=0.5$ since our guess of the solution is random. In the next time step, we need to make an assumption on $P_{00}(j, t)$. An intuitive assignment $P_{00}(j, t)=n_{0}(j, t)$ gives very good result. Unlike MOGA, where a chromosome mutates using the mutation matrix, here our Markov Chain Genetic Algorithm (MCGA) makes use of the transition probability matrix $P_{a b}(j, t)$ to move from one state to the next. The entire evolutionary computation of MCGA depends mainly on the ranking scheme used for the chromosomes.

\section{Experiments}

In order to evaluate the effectiveness of MCGA, we compare it with MOGAR (Mutation Only Genetic Algorithm by Row). We first choose a row to mutate according to the row mutation probability, same as MOGAR, but in the next step when we perform the locus mutation in this row we use the Markov Chain method. This gives a time dependent transition probability between the 0 and 1 states. We have performed numerous experiments to compare MCGA with MOGAR using mutation matrix. Note that this is a control comparison since we fix the first step to be the same.

We use the knapsack problem as an example [13]. The knapsack has a maximum weight volume, which we call the optimal number. We have 16 items weighting separately 1248163264128256512102420484096819216384 and 32768. Our goal is to find configuration of the items and put them into the knapsack so that we get as close to the limit defined by the maximum knapsack 
weight volume. In each chromosome, every locus represents an item, and 1 means we put it into the knapsack, while 0 means we don't. We set the optimal number (the weight volume of the knapsack) from 10000 to 11000, and get the result separately from MOGAR and MCGA, as shown in Fig.2. Each algorithm runs for 40 generations. Here we can see that MCGA has a higher probability of reaching the optimum than MOGAR, which very often get trapped in local optima.

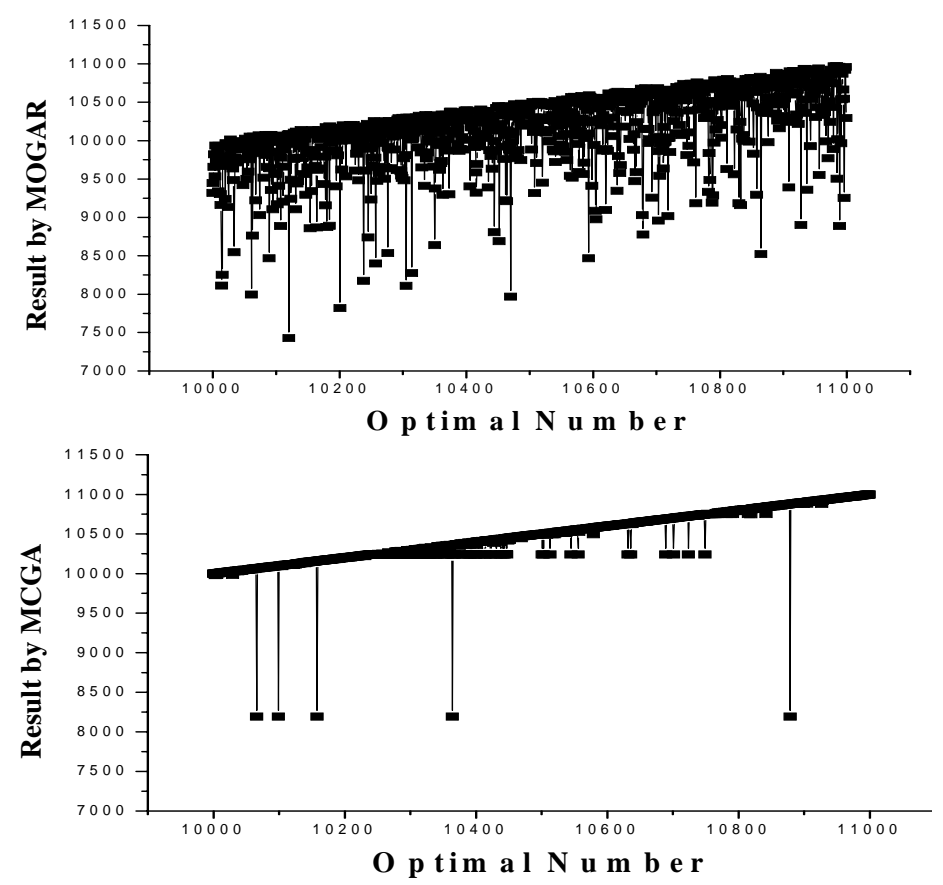

Fig.2. MOGAR and MCGA for the Knapsack Problem after 40 generations

Next we define a performance measure to compare these two algorithms. The first one is the "hit rate". For the "hit rate", we first introduce a "hit scale" by which we say that the result "hits" the optimum when it surpasses a critical threshold value. This threshold can be defined as a given percentage of the optimum. For example, if we set the threshold to be within 30\% of the optimum, then in our case with an optimal number 10000, we will say a particular solution of the knapsack problem has "hit" the optimum when its value lies between 7000 and 10000 . The "hit rate" is then defined as the number of hit result over the total number of tests performed and is between 0 for "never hitting" and 1 for "always hitting". In the context of our numerical work, we set the optimal number of the knapsack in the range between 10000 and 11000 , with a total number of tests being 1000 . We change the hit scale and the result is shown in Fig.3a. We can see that the hit rate of MCGA is closer to 1.0 than MOGAR with small hit scale. This indicates that MCGA can obtain more accurate results than MOGAR. 
The second performance measure used in the comparison of MOGA with MCGA address the number of generations in each algorithm. If we run these algorithms for a given number $(g)$ of generations, we can compute the average best fitness, which we call the "average fit rate", defined as

$$
\bar{F}(g)=\frac{1}{1000} \sum_{h=10000}^{11000} \frac{B(h, g)}{h}
$$

Here $B(h, g)$ is the fitness of the best chromosome in the $g$-th generation and with given optimum value of $h$. In a given test with $g$ generation and optimal number $h$, the fitness of the best chromosome will be $h$ if it hits the optimum value, otherwise it will be less. Therefore, the average fit rate will be smaller than one, unless the algorithm is perfect and find optimum every time for given $g$ and $h$. We have performed test for generation g less than 81. The results are shown in Fig.3b. We can see that MCGA has a better fit rate than MOGAR in general, especially in the beginning generations. This is consistent with the result for MCGA in Fig. 3a where we see that MCGA is more accurate than MOGAR. Furthermore, the result on fit rate indicates that MCGA can achieve more accurate results with less number of generations than MOGAR.

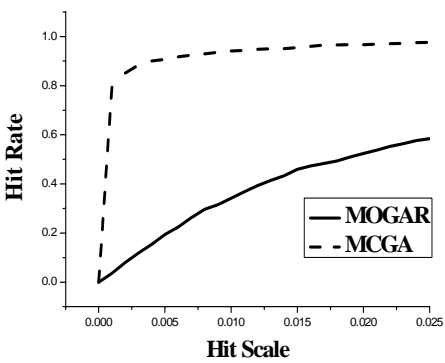

(a)

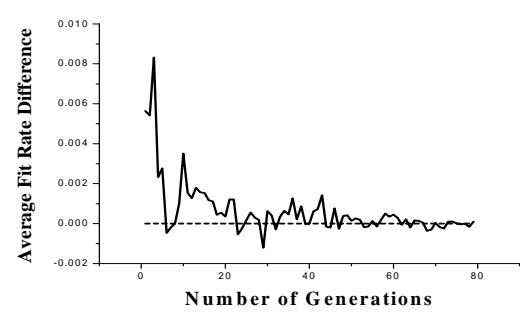

(b)

Fig.3. (a) Hit Rate and (b) Fit Rate for MOGAR and MCGA

\section{Conclusion}

We test our new genetic algorithm using a Markov Chain formulation of the mutation matrix, so that the direction of search is a guided search using a transition matrix based on a given ranking scheme of the chromosomes. This new algorithm (MCGA) makes transparent the fundamental assumption of the evolutionary process lies in the choice of ranking scheme. The important input for this guided search is the ranking scheme of the chromosomes. The simple ranking scheme used in this paper suggests that MCGA is better than the original formulation of MOGA, which makes use of past statistics to construct a mutation matrix. We compare a particular choice of MOGA using row first (MOGAR) and conclude 
that MCGA is in general better than MOGAR both in terms of accuracy (measured by hit rate) and in terms of speed (measured by fit rate) for the knapsack problem. We expect this superiority of MCGA over MOGA remains valid in other standard tests of the algorithms. Various combinations of these algorithm involve resource allocation, which have been addressed for MOGA, but not yet for MCGA. In future works, we will present the results on managing these combinations using intelligent resource allocation techniques $[9,11,14]$.

Acknowledgment K.Y. Szeto acknowledges the support of CERG grant 602506 and 602507.

\section{References}

[1] J. H. Holland, Adaptation in Natural and Artificial Systems. Ann Arbor, MI: University of Michigan Press, 1975

[2] D. E. Goldberg, Genetic Algorithms in Search, Optimization, and Machine Learning. AddisonWesley, Reading, MA, 1989.

[3] S. P. Li, K. Y. Szeto, “Crytoarithmetic problem using parallel Genetic Algorithms,” Mendl'99, Brno, Czech, 1999.

[4] K.Y. Szeto, K.H. Cheung, "Multiple time series prediction using genetic algorithms optimizer," Proceedings of the International Symposium on Intelligent Data Engineering and Learning, Hong Kong, IDEAL'98, 127-133, 1998.

[5] R. Jiang, K.Y. Szeto, Y.P. Luo, D.C. Hu, "Distributed parallel genetic algorithm with path splitting scheme for the large traveling salesman problems," Proceedings of Conference on Intelligent Information Processing, $16^{\text {th }}$ World Computer Congress 2000, Aug.21-25, 2000, Beijing, Ed. Z. Shi, B. Faltings, and M. Musen, Publishing House of Electronic Industry, 478-485, 2000.

[6] K.Y. Szeto, K.H. Cheung, S.P. Li, "Effects of dimensionality on parallel genetic algorithms," Proceedings of the $4^{\text {th }}$ International Conference on Information System, Analysis and Synthesis, Orlando, Florida, USA, Vol.2, 322 325, 1998.

[7] K.Y. Szeto, L.Y. Fong, "How adaptive agents in stock market perform in the presence of random news: a genetic algorithm approach,” LNCS/LNAI, Vol. 1983, Ed. K. S. Leung et al. SprigerVerlag, Heidelberg, 2000, IDEAL 2000, 505-510, 2000.

[8] Alex L.Y. Fong and K.Y. Szeto, Rule Extraction in Short Memory Time Series using Genetic Algorithms; European Physical Journal B Vol.20, 569-572(2001)

[9] King Loong Shiu and Kwok Yip Szeto; "Self-adaptive Mutation Only Genetic Algorithm: An Application on the Optimization of Airport Capacity Utilization”, IDEAL2008, November 2-5, at Daejeon, Korea LNCS5326,pp.428-435,2008, Springer-Verlag

[10] C.W. Ma and K.Y. Szeto, Locus Oriented Adaptive Genetic Algorithm: Application to the Zero/One Knapsack Problem, Proceeding of The 5th International Conference on Recent Advances in Soft Computing, RASC2004 Nottingham, UK. p.410-415, 2004.

[11] K. Y. Szeto and J. Zhang, in I. Lirkov, S. Margenov, and J. Wasniewski (Eds.): LSSC 2005 June Sozopol, LNCS3743, pp.189-196, 2006. Springer-Verlag

[12] N.L. Law and K.Y. Szeto; Adaptive Genetic Algorithm with Mutation and Crossover Matrices; Proceeding of the $12^{\text {th }}$ International Joint Conference on Artificial Intelligence (IJCAI-07) January 6 - 12, 2007 (Volume II) Theme: Al and Its Benefits to Society, Published by International Joint Conferences on Artificial Intelligence, IJCAI-07. Hyderabad, India, pp 2330 - 2333

[13] V. Gordon, A. Bohm, and D. Whitley, A Note on the Performance of Genetic Algorithms on Zero-One Knapsack Problems. Proceedings of the 9th Symposium on Applied Computing (SAC'94), Genetic Algorithms and Combinatorial Optimization, Phoenix, Az, pp 194-195(1994)

[14] Kwok Yip Szeto, Jiang Rui, A quasi-parallel realization of the Investment Frontier in Computer Resource Allocation Using Simple Genetic Algorithm on a Single Computer. LNCS 2367, 6th International Conference, PARA 2002, Espoo, Finland, June 15-18, 2002 pp.116-126. SpringerVerlag. 